\title{
Short communication: Oral and intranasal administration of a modified-live Salmonella Dublin vaccine in dairy calves: Clinical efficacy and serologic response
}

\author{
Kevin J. Cummings, ${ }^{1,2 *}$ Lorraine D. Rodriguez-Rivera, ${ }^{2}$ Michael B. Capel, ${ }^{3}$ Shelley C. Rankin, ${ }^{4}$ \\ and Daryl V. Nydam ${ }^{1}$ \\ ${ }^{1}$ Department of Population Medicine and Diagnostic Sciences, College of Veterinary Medicine, Cornell University, Ithaca, NY 14853 \\ ${ }^{2}$ Department of Veterinary Integrative Biosciences, College of Veterinary Medicine \& Biomedical Sciences, Texas A\&M University, \\ College Station 77843 \\ ${ }^{3}$ Perry Veterinary Clinic, PLLC, Perry, NY 14530 \\ ${ }^{4}$ Department of Pathobiology, School of Veterinary Medicine, University of Pennsylvania, Philadelphia 19104
}

\section{ABSTRACT}

Our objectives were to evaluate the clinical efficacy of oral and intranasal administration of a commercial modified-live Salmonella Dublin vaccine in dairy calves and to determine the serologic response associated with these extralabel routes of administration. We conducted a randomized field trial with calves from a New York dairy farm following an outbreak of Salmonella Dublin. A total of 399 Holstein calves were allocated by pen to 3 treatment groups: oral vaccination, intranasal vaccination, and an unvaccinated control group. Administration of the vaccine through oral and intranasal routes did not have a significant effect on pneumonia incidence risk or weight gain; however, calves vaccinated orally and intranasally had lower mortality risk as compared with control calves. Among calves tested using a Salmonella Dublin ELISA, vaccination did not induce an increase in antibody production relative to control calves, indicating that oral and intranasal administration will not hinder diagnosis based on this assay.

Key words: dairy calf, field trial, Salmonella Dublin, vaccine

\section{Short Communication}

Salmonella enterica serotype Dublin is an important cause of salmonellosis among dairy cattle in the United States and other areas of the world (Izzo et al., 2011; Nielsen and Dohoo, 2013; Valenzuela et al., 2017). Illness occurs primarily in calves under 2 mo of age, with a

Received April 6, 2018.

Accepted December 7, 2018.

*Corresponding author: kjc39@cornell.edu predominant clinical manifestation of respiratory rather than gastrointestinal disease. This serotype is hostadapted to cattle, and infection may result in chronic, subclinical carriers that continuously or intermittently shed high numbers of organisms into the environment. Carrier animals play a key role in maintaining infection in dairy herds via shedding of Salmonella Dublin in feces, milk, and colostrum (Holschbach and Peek, 2018). Salmonella Dublin also poses a threat to public health, with a predilection for causing invasive disease with relatively high case fatality among human patients (Harvey et al., 2017).

Stimulating a protective immune response against Salmonella Dublin through vaccination would be a valuable control measure to be used in conjunction with other farm management practices. Modified-live Salmonella vaccines induce humoral and cell-mediated immune responses, both of which are needed for effective protection against this pathogen (Mastroeni et al., 2001). A commercial modified-live Salmonella Dublin vaccine (EnterVene-d; Boehringer Ingelheim Vetmedica Inc., Duluth, GA) is approved in the United States for subcutaneous administration $(2 \mathrm{~mL})$ in calves that are at least 2 wk of age, with a second $2-\mathrm{mL}$ dose recommended for administration 12 to $16 \mathrm{~d}$ after the first vaccination. In the field, extralabel routes of administration of this vaccine are also used to help control salmonellosis caused by Salmonella Dublin, to avoid potential adverse reactions associated with parenteral administration (Habing et al., 2011). However, the clinical effectiveness and serologic implications of extralabel administration are poorly understood. Thus, the objectives of our study were to evaluate the clinical efficacy of oral and intranasal administration of the modified-live Salmonella Dublin vaccine in dairy calves and to determine the serologic response associated with these routes of administration. 
This study was a randomized field trial that included Holstein calves allocated to 3 treatment groups: oral vaccination with the modified-live Salmonella Dublin vaccine, intranasal vaccination with the modified-live Salmonella Dublin vaccine, and no vaccination (control group). Each calf pen on the farm typically housed 22 calves, and 6 pens were allocated to each of the 3 treatment groups through cluster randomization. Therefore, the experimental unit was the pen, and 18 pens were sequentially filled with calves and included in this study. These 18 pens were assigned to treatment group based on a randomized block design in which pens were blocked on time using a random number generator. Calves born between October 30, 2013, and July 22, 2014, were enrolled in the study. Two trained calf personnel were responsible for monitoring clinical outcomes, recording data, and collecting samples, and they were blinded to group assignments. At least 1 of the 2 was present $7 \mathrm{~d} / \mathrm{wk}$, and both were present $5 \mathrm{~d} /$ wk. This study was approved by the Texas A\&M University Institutional Animal Care and Use Committee, and informed consent was obtained from the owner of the participating dairy farm. The extralabel routes of administration were implemented by the veterinarian of record at the study farm, who maintained a valid veterinarian-client-patient relationship. The veterinarian and study farm owner chose these administration routes in an effort to minimize potential adverse reactions while remaining in compliance with relevant laws and standards of practice.

The trial was conducted on a heifer-raising facility in a major dairy-producing region of New York. This facility raises heifers from its own dairy herd as well as heifers from a neighboring dairy herd, with no mixing of animals from the 2 sources. Only calves from the neighboring dairy herd were included in our study. The study herd was conventionally managed and included over 1,000 lactating cows. Calves were born in a maternity pen and remained for 1 to $6 \mathrm{~h}$, depending on employee attentiveness. Within $2 \mathrm{~h}$ of birth, $3.8 \mathrm{~L}$ of pasteurized colostrum were tube fed. The study herd had a history of Salmonella Dublin infection, and the trial was initiated following an outbreak that began in fall 2012 and resulted in morbidity (respiratory disease) and mortality among young calves. Salmonella Dublin was isolated from clinically ill calves and from necropsied calves during this outbreak. Intranasal use of the modified-live Salmonella Dublin vaccine was initiated shortly after the outbreak began and continued until the start of the trial.

Calves arrived at the heifer raiser at approximately $3 \mathrm{~d}$ of age and were housed in pens with a base of sawdust bedding and straw added on top as needed. Calves were initially fed acidified waste milk ad libitum up to $18 \mathrm{~L} / \mathrm{d}$ and calf starter was offered immediately; waste milk was acidified using $85 \%$ formic acid, and the $\mathrm{pH}$ was maintained at 4.1 to 4.3 . During the period between 5 and 7 wk of age, the volume of waste milk fed to calves was gradually reduced to $2 \mathrm{~L} / \mathrm{d}$. At $7 \mathrm{wk}$ of age, calves were no longer offered waste milk. Calves were switched to a grower pellet at approximately 6 wk of age. At the time of transport from the home farm to the heifer raiser, calves were administered an intranasal vaccine against viral respiratory pathogens (INFORCE 3; Zoetis, Parsippany, NJ). Between 2 and 3 wk of age, calves were subcutaneously administered a tetanus toxoid and Clostridium perfringens vaccine (Bar-Vac CD/T; Boehringer Ingelheim Vetmedica Inc.). No other vaccines unrelated to the study were administered to calves.

The oral and intranasal vaccine protocols used in our study were designed to closely mimic the protocols used for extralabel administration of the modified-live Salmonella Dublin vaccine in the field. Calves in the oral vaccine group were orally administered $4 \mathrm{~mL}$ of vaccine mixed with $16 \mathrm{~mL}$ of antacid solution (containing $5 \%$ sodium bicarbonate, $5 \%$ magnesium carbonate, and $5 \%$ magnesium trisilicate) at 4 and $18 \mathrm{~d}$ of age. Calves were allowed to suckle the mixture directly from the syringe; otherwise, the mixture was carefully administered into the oropharynx. Calves in the intranasal vaccine group were administered $2 \mathrm{~mL}$ of vaccine into one of the nares at 4 and $18 \mathrm{~d}$ of age. Calves in the control group were not administered the modified-live Salmonella Dublin vaccine; a sham vaccine was not used because those farm personnel responsible for vaccine administration were distinct from the calf workers blinded to group assignments.

The BW of each calf was measured at pen entry and again at weaning to calculate ADG. Calves were monitored daily for clinical signs compatible with pneumonia or enteritis, including dyspnea, coughing, diarrhea, fever [body temperature $\geq 39.44^{\circ} \mathrm{C}\left(103.0^{\circ} \mathrm{F}\right)$ ], and depression. A clinical presentation of either (1) dyspnea or (2) coughing combined with either fever or depression was considered a case of pneumonia, and a clinical presentation of diarrhea combined with either fever or depression was considered a case of enteritis. Fecal samples were collected from any calf diagnosed with pneumonia or enteritis. Approximately $10 \mathrm{~g}$ of fecal matter was placed into a Para-Pak C \& S bottle (Meridian Bioscience Inc., Cincinnati, $\mathrm{OH}$ ) for transport. Each sample was labeled with the date of sampling, calf identification number, and pen number. Samples 
were shipped overnight to the research laboratory at Texas A\&M University (College Station, TX) for bacteriologic culture to isolate Salmonella.

Blood samples were collected from calves at 2 time points; our aim was to collect samples from a subset of calves at 10 wk of age (i.e., the first 10 calves entering each pen) and from all calves at 10 mo of age. Approximately $4 \mathrm{~mL}$ of blood was collected from the jugular vein using an aseptic technique and placed into a redtop blood collection tube (Becton, Dickinson and Company, Franklin Lakes, NJ). Samples were maintained at approximately $4^{\circ} \mathrm{C}$ during overnight shipment to the Animal Health Diagnostic Center at Cornell University (Ithaca, NY). Each sample was labeled with the date of sampling, calf identification number, and pen number. Antibody testing of blood samples was performed using a Salmonella Dublin ELISA.

Standard bacteriologic culture methods were used to isolate Salmonella from fecal samples as previously described (Cummings et al., 2016), with the exception that we used Xylose Lysine Tergitol 4 and Brilliant Green selective media (Northeast Laboratory Services, Winslow, ME). Presumptive Salmonella isolates were confirmed by amplification and detection of the invA gene using PCR (Kim et al., 2007). Confirmed Salmonella isolates were sent to the Clinical Microbiology Laboratories at the University of Pennsylvania Veterinary Hospital for serotyping, using the xMAP Salmonella Serotyping Assay (Luminex, Austin, TX) as previously described (Cummings et al., 2016). Antimicrobial susceptibility of confirmed Salmonella isolates was determined using a broth microdilution method. Minimum inhibitory concentrations were established for each isolate against the National Antimicrobial Resistance Monitoring System gram-negative panel of 14 antimicrobial agents (Sensititer; TREK Diagnostic Systems, Cleveland, $\mathrm{OH}$ ): amoxicillin/clavulanic acid, ampicillin, azithromycin, cefoxitin, ceftiofur, ceftriaxone, chloramphenicol, ciprofloxacin, gentamicin, nalidixic acid, streptomycin, sulfisoxazole, tetracycline, and trimethoprim/sulfamethoxazole.

Serum antibody testing was performed at the Animal Health Diagnostic Center using a Salmonella Dublin ELISA kit (PrioCHECK Salmonella Antibody ELISA Dublin; Thermo Fisher Scientific, Waltham, MA). The result for each sample was expressed as percent positivity, which was calculated according to a formula that relates antibody value of a sample to the value of the assay's positive control. Samples were reported as positive when the percent positivity was $\geq 35$. A positive result indicates the presence of antibodies against Salmonella Dublin, suggesting prior exposure.
Data were imported into a commercial statistical software program (SAS, version 9.4; SAS Institute Inc., Cary, NC) for variable coding and analysis. Descriptive analysis of all variables was performed. Disease incidence risk and mortality risk were calculated over the entire 10-mo period of clinical monitoring during the study. Poisson regression models were used to determine if treatment group was associated with risk of clinical disease and mortality, with these outcomes being converted to pen-level measurements. Poisson regression models were also used to determine if treatment group was associated with risk of positive fecal culture and serologic status among tested animals, with these outcomes again being converted to pen-level measurements. A linear regression model was used to determine if treatment group was associated with mean ADG per pen. The generalized estimating equations method was used in all of these models to account for the clustering of pens by block. The ELISA percent positivity values at $10 \mathrm{wk}$ and $10 \mathrm{mo}$ of age were compared across treatment groups using Friedman's test (nonparametric 2 -way ANOVA). For all analyses, $P$-values $<0.05$ were considered significant.

A total of 399 calves were enrolled in the study (130 in oral group, 137 in intranasal group, and 132 in control group), and the median number of calves per pen was 22 (range, 21 to 25). Pneumonia was identified in $91(22.8 \%)$ calves, at a median age of $40 \mathrm{~d}$ (range $=10-106 \mathrm{~d}$; interquartile range $=28-56 \mathrm{~d}$ ). Enteritis was not reported during the study. Seventeen (4.3\%) calves died, at a median age of $69 \mathrm{~d}$ (range $=9-91 \mathrm{~d}$; interquartile range $=40-76 \mathrm{~d})$. Most of these $(14 / 17)$ were calves in which pneumonia had been reported within the previous 2 mo. The median number of calf deaths per pen was 1 (range $=0-4)$. The total number of calf deaths per each of the 6 pens was $0,0,1,1,1$, and 1 for the oral group, $0,0,0,0,1$, and 1 for the intranasal group, and $0,0,1,3,3$, and 4 for the control group. Seven of 91 pneumonia cases and 1 of 17 deaths occurred before administration of the second vaccine dose at $18 \mathrm{~d}$ of age. The mean ADG was $0.87 \mathrm{~kg}$. $(95 \%$ $\mathrm{CI}=0.85-0.88 \mathrm{~kg})$. Fecal samples were collected from $112(28.1 \%)$ calves for bacteriologic culture; of these, 12 $(10.7 \%)$ were found to be positive for Salmonella, with serotypes Dublin (7/12) and Cerro (5/12) identified. All Salmonella Dublin isolates were multidrug resistant, displaying resistance to amoxicillin/clavulanic acid, ampicillin, cefoxitin, ceftiofur, ceftriaxone, chloramphenicol, streptomycin, sulfisoxazole, and tetracycline. All Salmonella Cerro isolates were pan-susceptible.

Pneumonia incidence risk over the course of our study was $18.5 \%(24 / 130)$ in the oral group, $27.7 \%$ 
$(38 / 137)$ in the intranasal group, and $22.0 \%(29 / 132)$ in the control group $(P=0.4)$. Mortality occurred in $3.1 \%(4 / 130)$ of calves in the oral group, $1.5 \%(2 / 137)$ of calves in the intranasal group, and $8.3 \%(11 / 132)$ of calves in the control group. Calves vaccinated orally $[$ risk ratio $(\mathbf{R R})=0.4 ; 95 \% \mathrm{CI}=0.2-0.9 ; P=0.02$ ] and intranasally $(\mathrm{RR}=0.2 ; 95 \% \mathrm{CI}=0.05-0.6 ; P$ $=0.004)$ had lower mortality risk as compared with control calves. When including only calf deaths that followed pneumonia, calves vaccinated orally $(\mathrm{RR}=$ $0.2 ; 95 \% \mathrm{CI}=0.06-0.8 ; P=0.02)$ and intranasally $(\mathrm{RR}=0.2 ; 95 \% \mathrm{CI}=0.05-0.8 ; P=0.02)$ likewise had lower mortality risk. Mean ADG was $0.88 \mathrm{~kg}(95 \%$ CI $=0.85-0.91 \mathrm{~kg})$ in the oral group, $0.85 \mathrm{~kg}(95 \% \mathrm{CI}$ $=0.82-0.88 \mathrm{~kg})$ in the intranasal group, and $0.87 \mathrm{~kg}$ $(95 \% \mathrm{CI}=0.83-0.91 \mathrm{~kg})$ in the control group $(P=$ 0.7). Among calves that were sampled for fecal culture, the risk of testing positive for Salmonella did not differ significantly by treatment group.

Blood samples were collected from 156 (39.1\%) calves for Salmonella Dublin ELISA testing at $10 \mathrm{wk}$ of age; of these, $13(8.3 \%)$ yielded a positive result. Blood samples were collected from $369(92.5 \%)$ calves for ELISA testing at $10 \mathrm{mo}$ of age, and $33(8.9 \%)$ of these were positive. Of the 149 calves tested at both time points, $125(83.9 \%)$ were negative at both, 11 (7.4\%) were positive at $10 \mathrm{wk}$ but negative at $10 \mathrm{mo}$, $12(8.1 \%)$ were negative at $10 \mathrm{wk}$ but positive at $10 \mathrm{mo}$, and $1(0.7 \%)$ was positive at both time points. Among calves found to be positive via fecal culture, the median percent positivity was 55 (range $=-12$ to 154 ) at 10 wk and -2 (range $=-14$ to 72 ) at 10 mo.

Risk of positive serologic status did not differ significantly by treatment group among ELISA-tested calves at either 10 wk or 10 mo of age. Similarly, median percent positivity values did not vary significantly by treatment group among ELISA-tested calves at either 10 wk or 10 mo of age (Table 1 ).

In our study, administration of the modified-live Salmonella Dublin vaccine through oral and intranasal routes did not have a significant effect on pneumonia incidence risk or weight gain among dairy calves; however, mortality risk was lower among calves vaccinated by either route. This effect was evident when consider- ing all deaths as well as deaths following pneumonia, suggesting that vaccination via the oral and intranasal routes may reduce disease severity in calves. Reduction of calf mortality associated with Salmonella Dublin outbreaks on dairy farms would help mitigate economic losses caused by this disease. Nevertheless, it must be emphasized that we measured calf mortality from all causes, and the deaths observed cannot necessarily be attributed to infection with Salmonella Dublin. Tissue samples were not collected from calves that died for either histopathologic or bacteriologic analysis. In a previous randomized field trial, investigators also found that oral administration of the modified-live Salmonella Dublin vaccine did not have a significant effect on disease incidence or growth among dairy calves (Habing et al., 2011). However, in that study, mortality was relatively low overall and was comparable in vaccinated calves and control calves. That trial was also initiated following a farm outbreak of Salmonella Dublin among dairy calves, but the incidence of salmonellosis had declined by the time of study onset; only $4 \%$ of 161 collected fecal samples were found to be culturepositive for Salmonella, and no isolates were identified as serotype Dublin (Habing et al., 2011). In another experimental study, researchers demonstrated that a modified-live Salmonella Dublin vaccine administered subcutaneously to dairy calves was associated with reduced mortality relative to control calves (Selim et al., 1995).

The apparent prevalence of fecal Salmonella shedding among clinically ill calves in our study (11\%) was lower than anticipated, although the majority of positive calves were confirmed to be shedding multidrugresistant Salmonella Dublin. Fecal culture status did not vary significantly by treatment group, suggesting that vaccination does not reduce the likelihood of dairy farm environmental contamination or the risk to public health. However, fecal culture has poor sensitivity for detecting the presence of Salmonella Dublin (Nielsen et al., 2004a; Baggesen et al., 2007). We recognize that some positive calves were presumably missed by culturing, particularly because we did not perform serial testing. In addition, other clinical specimens might have been more valuable for identifying Salmonella Dublin,

Table 1. Percent positivity [median (range)] among dairy calves tested using a Salmonella Dublin ELISA by age and treatment group (defined by modified-live Salmonella Dublin vaccine status) ${ }^{1}$

\begin{tabular}{lccc}
\hline Age & $\begin{array}{c}\text { Oral } \\
\text { vaccine group }\end{array}$ & $\begin{array}{c}\text { Intranasal } \\
\text { vaccine group }\end{array}$ & $\begin{array}{c}\text { Control } \\
\text { group }\end{array}$ \\
\hline $10 \mathrm{wk}$ & $-11(-13$ to 125$)$ & $-10(-13$ to 195$)$ & $-11(-13$ to 70$)$ \\
$10 \mathrm{mo}$ & $-1.5(-11$ to 126$)$ & $-3.5(-14$ to 160$)$ & $0(-11$ to 194$)$ \\
\hline
\end{tabular}

${ }^{1}$ Samples were reported as positive when the percent positivity was $\geq 35$. 
including blood, transtracheal wash, and lung tissue samples. Conversely, we cannot rule out the possibility of other infectious agents as the cause of disease in some calves. Other causes of dairy calf pneumonia are numerous and include bacterial, viral, and parasitic agents (Divers and Peek, 2018).

Among ELISA-tested calves in our study, administration of the modified-live Salmonella Dublin vaccine through oral and intranasal routes did not induce an increase in antibody production relative to control calves. Any reduction in disease severity in vaccinated calves, if indeed attributable to the vaccine, was presumably enabled by an enhanced cell-mediated immune response (Mastroeni et al., 2001). This result also suggests that oral and intranasal administration of the vaccine will not complicate the interpretation of Salmonella Dublin ELISA results and thus will not impede diagnosis based on this assay. In addition, our data implied no differences in natural exposure to Salmonella Dublin among treatment groups; we can infer that our evaluation of clinical parameters for vaccine efficacy was not confounded by differential pathogen exposure across these groups.

Our ability to evaluate the relationship between vaccination and risk of Salmonella Dublin carrier status is limited. Animals were ELISA tested a maximum of two times, with a targeted interval between tests of approximately $230 \mathrm{~d}$. Most (20/33) animals that were Salmonella Dublin antibody-positive at 10 mo of age were not tested at $10 \mathrm{wk}$; thus, their prior serologic status is unknown. Of the 149 calves that were ELISA tested at both 10 wk and 10 mo of age, only 1 was found to be positive at both time points. Other investigators have used various diagnostic protocols to establish presumptive carrier status, including 2 serum ELISA tests performed $60 \mathrm{~d}$ apart (Spier et al., 1990), 3 serum ELISA tests over a period of $120 \mathrm{~d}$ (Smith et al., 1992), and 4 tests (ELISA tests and fecal cultures were performed in parallel) over a period of $270 \mathrm{~d}$ (Nielsen et al., 2004b). Regardless, recent work indicates that repeated antibody testing in Salmonella Dublin-infected dairy herds may not be a reliable method for predicting fecal shedding status (Nielsen, 2013). In that study, the risk of fecal shedding among cattle with persistently high Salmonella Dublin antibody levels was not statistically different than the risk among cattle with either recently increased or fluctuating antibody levels.

In addition to being efficacious, a vaccination protocol needs to be practical and cost-effective and have a demonstrated safety record to merit widespread adoption. Farm personnel considered the intranasal route of vaccine administration to be straightforward and convenient. In contrast, the oral route was perceived as cumbersome and inefficient; the need to mix the vaccine with antacid solution beforehand added to the difficulty involved. We observed no apparent adverse reactions associated with either the oral or intranasal route of administration during the course of the study. Based on the experience of farm personnel and the comparable safety of the 2 administration routes in our study, the intranasal route of modified-live Salmonella Dublin vaccine administration appears to be preferable over the oral route. It is also important to note that the concentration of organisms in the modified-live Salmonella Dublin vaccine may simply be insufficient for generating a robust immune response following oral use. Conferment of passive immunity to calves via subcutaneous administration of this vaccine to late-gestation cows may also be a useful approach, although clinical efficacy of this protocol has not been established (Smith et al., 2015).

\section{ACKNOWLEDGMENTS}

We thank the dairy farm personnel who monitored clinical outcomes, recorded data, and collected samples over the duration of the study. Boehringer Ingelheim Vetmedica Inc. (Duluth, GA) provided the vaccine to the veterinarian of record at the study farm and paid for the diagnostic testing.

\section{REFERENCES}

Baggesen, D. L., L. R. Nielsen, G. Sorensen, R. Bodker, and A. K Ersboll. 2007. Growth inhibitory factors in bovine faeces impairs detection of Salmonella Dublin by conventional culture procedure. J. Appl. Microbiol. 103:650-656.

Cummings, K. J., L. D. Rodriguez-Rivera, M. K. Grigar, S. C. Rankin, B. T. Mesenbrink, B. R. Leland, and M. J. Bodenchuk. 2016. Prevalence and characterization of Salmonella isolated from feral pigs throughout Texas. Zoonoses Public Health 63:436-441.

Divers, T. J., and S. F. Peek. 2018. Rebhun's Diseases of Dairy Cattle. 3rd ed. Saunders Elsevier, St. Louis, MO.

Habing, G. G., L. M. Neuder, W. Raphael, H. Piper-Youngs, and J. B. Kaneene. 2011. Efficacy of oral administration of a modifiedlive Salmonella Dublin vaccine in calves. J. Am. Vet. Med. Assoc. 238:1184-1190.

Harvey, R. R., C. R. Friedman, S. M. Crim, M. Judd, K. A. Barrett, B. Tolar, J. P. Folster, P. M. Griffin, and A. C. Brown. 2017. Epidemiology of Salmonella enterica serotype Dublin infections among humans, United States, 1968-2013. Emerg. Infect. Dis. 23:1493-1501.

Holschbach, C. L., and S. F. Peek. 2018. Salmonella in dairy cattle. Vet. Clin. North Am. Food Anim. Pract. 34:133-154.

Izzo, M., V. Mohler, and J. House. 2011. Antimicrobial susceptibility of Salmonella isolates recovered from calves with diarrhoea in Australia. Aust. Vet. J. 89:402-408.

Kim, J. S., G. G. Lee, J. S. Park, Y. H. Jung, H. S. Kwak, S. B. Kim, Y. S. Nam, and S. T. Kwon. 2007. A novel multiplex PCR assay for rapid and simultaneous detection of five pathogenic bacteria: Escherichia coli 0157:H7, Salmonella, Staphylococcus aureus, Listeria monocytogenes, and Vibrio parahaemolyticus. J. Food Prot. 70:1656-1662 
Mastroeni, P., J. A. Chabalgoity, S. J. Dunstan, D. J. Maskell, and G. Dougan. 2001. Salmonella: Immune responses and vaccines. Vet. J. 161:132-164.

Nielsen, L. R. 2013. Salmonella Dublin faecal excretion probabilities in cattle with different temporal antibody profiles in 14 endemically infected dairy herds. Epidemiol. Infect. 141:1937-1944.

Nielsen, L. R., and I. Dohoo. 2013. Time-to-event analysis of predictors for recovery from Salmonella Dublin infection in Danish dairy herds between 2002 and 2012. Prev. Vet. Med. 110:370-378.

Nielsen, L. R., Y. H. Schukken, Y. T. Grohn, and A. K. Ersboll. 2004b. Salmonella Dublin infection in dairy cattle: Risk factors for becoming a carrier. Prev. Vet. Med. 65:47-62.

Nielsen, L. R., N. Toft, and A. K. Ersboll. 2004a. Evaluation of an indirect serum ELISA and a bacteriological faecal culture test for diagnosis of Salmonella serotype Dublin in cattle using latent class models. J. Appl. Microbiol. 96:311-319.

Selim, S. A., J. S. Cullor, B. P. Smith, P. Blanchard, T. B. Farver, R. Hoffman, G. Dilling, L. D. Roden, and B. Wilgenburg. 1995. The effect of Escherichia coli J5 and modified live Salmonella Dublin vaccines in artificially reared neonatal calves. Vaccine 13:381-390.
Smith, B. P., J. K. House, G. W. Dilling, L. D. Roden, and S. J. Spier. 1992. Identification of Salmonella Dublin carrier cattle. Pages 225230 in Proceedings of the International Symposium on Salmonella and Salmonellosis, Ploufragan, France. I3S, Ploufragan, France.

Smith, G. W., F. Smith, S. Zuidhof, and D. M. Foster. 2015. Short communication: Characterization of the serologic response induced by vaccination of late-gestation cows with a Salmonella Dublin vaccine. J. Dairy Sci. 98:2529-2532.

Spier, S. J., B. P. Smith, J. W. Tyler, J. S. Cullor, G. W. Dilling, and L. Da Pfaff. 1990. Use of ELISA for detection of immunoglobulins G and M that recognize Salmonella Dublin lipopolysaccharide for prediction of carrier status in cattle. Am. J. Vet. Res. 51:1900-1904

Valenzuela, J. R., A. K. Sethi, N. A. Aulik, and K. P. Poulsen. 2017. Antimicrobial resistance patterns of bovine Salmonella enterica isolates submitted to the Wisconsin Veterinary Diagnostic Laboratory: 2006-2015. J. Dairy Sci. 100:1319-1330. 